\title{
A comprehensive model for char particle conversion in environments containing $\mathrm{O}_{2}$ and $\mathrm{CO}_{2}$
}

\author{
Nils Erland L. Haugen ${ }^{1,2}$, Reginald E. Mitchell ${ }^{3}$ and Matthew B. Tilghman ${ }^{3}$ \\ ${ }^{1}$ SINTEF Energy Research, NO-7465 Trondheim, Norway \\ ${ }^{2}$ Department of Energy and Process Engineering, Norwegian University of Science and \\ Technology, Kolbjørn Hejes vei 1B, NO-7491 Trondheim, Norway \\ ${ }^{3}$ Department of Mechanical Engineering, Stanford University, USA
}

\begin{abstract}
In this work, conversion of char is studied in a simulation code that includes a simplified heterogeneous adsorption-desorption reaction mechanism for char chemical reactivity and uses GRI-Mech 3.0 as the chemical kinetic mechanism that describes the impact of homogeneous reactions. Besides sub-models for the consequences of chemical reactions, a mode of char particle conversion sub-model is included that describes how particle size and apparent density vary with mass loss as well as a radiation sub-model that describes the radiant exchange of energy between the char particle and surrounding particles and walls. The code is transient and zero dimensional in space, and designed to be used both as a stand-alone gasification/combustion code and as a sub-model for heterogeneous reactions of solid particles in a CFD code.
\end{abstract}

Keywords: combustion; gasification; char

\section{Introduction}

The coal conversion process consists of a fast devolatilization phase (which includes particle drying during heat-up) followed by a slower char gasification/combustion phase. Single particle char conversion can be modeled using transient zero dimensional models (which have no spatial discretization), which is done by Samuilov et al. [1], for example, and elsewhere [2, 3]. Recently Qiao et al. (2012) [4] have taken this approach a step further by accounting not only for a single particle but for the effect of a cloud of particles on the gas phase temperature. For particle clouds, unless the particle 
number density is very low, the gas phase composition, temperature and mass may be significantly modified by effects associated with heterogeneous reactions. Clouds of reactive particles, typically in gasifiers, have also been studied previously. Govind and Shah [5] used a plug flow reactor to simulate a Texaco gasifier, including pyrolysis, gas phase combustion and solid phase gasification. One step kinetics were used both for the homogeneous and heterogeneous chemical reactions. Radiative transfer between particles and the gas was included by assuming the gas phase to be optically thick. $\mathrm{Ni}$ and Williams [6] studied pyrolysis, combustion and gasification in a Shell gasifier. They used a simplified set of heterogeneous and homogeneous chemical reactions, where some of the homogeneous reactions were assumed to be in equilibrium. In their approach, the reactor was assumed to consist of two regions, which were simulated as two zero dimensional reactors. In the first region only devolatilization and oxidation was considered while the second region considered gasification and reduction. In the work by Vamvuka et al. [7], a one-dimensional model of a plug flow reactor was used to simulate a generic entrained flow gasifier. Here a simplified kinetics model was used for the heterogeneous reactions, which were assumed to be confined to the particle outer surface (i.e. no pore reactions). The homogeneous chemistry was assumed to be in equilibrium at any given time. Later Liu et al. [8] extend the work of Vamvuka et al. and $\mathrm{Ni}$ and Williams by accounting for pore diffusion and variable surface area of the char particles.

The purpose of the current paper is to extend the works described above by developing a model that also takes into account 1) detailed homogeneous and heterogeneous chemical kinetics, 2) particle-wall and particle-particle radiation and 3) variations in particle size and apparent density. The model described here is based on a constant-pressure, fixed-mass reacting system (CPFM), while several of the previous models assumes a plug flow reactor configuration (PFR). In the PFR approach the dependent variables vary along a spatial dimension while in the CPFM approach the dependent variables vary in time. An advantage of the PFR approach is that the velocity field is a part of the solution, while an advantage of the CPFM approach is that it can be used directly as a sub-model for the reactive particles in a CFD simulation.

Depending on the ratio between the reactive and the diffusive time scales of a char particle, $\tau_{r}$ and $\tau_{D}$, respectively, char conversion will proceed in one of three possible different regimes. In the zone I conversion regime (in which $\tau_{r} \gg \tau_{D}$, rendering mass loss rates limited by chemical reaction rates), 
a particle reacts more or less uniformly throughout its volume. Its size is relatively unchanged and its apparent density varies proportionally with mass loss. In the zone III conversion regime $\left(\tau_{r} \ll \tau_{D}\right.$, rendering mass loss rates limited by the rates of reactant diffusion to the outer surfaces of particles), a particle reacts primarily at its periphery. Its apparent density is relatively unchanged and its diameter varies to the one-third power with mass loss. In between these two extremes is the zone II regime in which gradients of reactants, products and apparent density exist inside the particle. Due to the competing effects of pore diffusion and heterogeneous reactions, this is the most complicated regime to model. In attempts to account for particles that have only been partially penetrated by reactive gases, approaches that discretize particles into spherical shells have been developed [13, 14]. However, resolving the internal particle gradients is CPU intensive, which prohibits this strategy for CFD simulations, for example, when a large number of particles are considered. The intensive computational effort is avoided if the modelling strategy of Thiele [20] is employed, wherein an effectiveness factor is used to account for incomplete penetration of the reactive gases. This is the approach employed in this work.

In this paper, a model is developed that predicts the temporal variations in the mass, apparent density and diameter of a porous char particle when it is exposed to a hot gaseous environment composed of reactive gases $\left(\mathrm{O}_{2}\right.$ and $\mathrm{CO}_{2}$ ). Account is made for detailed, finite-rate heterogeneous chemical reactions that consume the carbonaceous material on both internal and external particle surfaces, convective and conductive heat transfer between the particle and the gaseous environment, and inter-particle radiation exchange, assuming that the particle is in a cloud of reacting particles. Account is also made for the detailed finite rate homogeneous chemical reactions that occur between desorbed species and reactive gas phase species.

The paper focuses on what is called the stand-alone model, a model in which calculations are made on a single particle and all other particles are assumed to behave in a similar fashion. Furthermore, the gas-particle mixture is assumed to be uniform. The model is suitable as a sub-model for CFD simulations of heterogeneous reacting particles.

\section{Model description}

In the model, particles are assumed to be spherical and uniform in composition and morphology. In addition, ash in the particle is assumed to be 
uniformly distributed throughout the particle volume. Furthermore, the assumption is also made that there is no ash vaporization or reactions that result in any ash component leaving the particle.

Let $V$ define the volume enclosed by the surface $S$, containing a gas mixture with a constant number of embedded char particles, $N_{p}$. Let the surface $S$ be impermeable such that there is no mass flux across it, and let it be flexible such that the volume $V$ is allowed to change in order to keep the gas pressure constant. The total mass inside $S$ is therefore constant and equal to $m=m_{p} N_{p}+m_{g}$ where $m_{p}$ is the mass of each particle and $m_{g}$ is the mass of the gas. The gas density is given by $\rho_{g}=m_{g} /\left(V-N_{p} V_{p}\right)$, where $V_{p}$ is the volume of a single particle, while the particle number density is $n_{p}=N_{p} / V$.

Due to reactions between the gas and the solid phases there will be exchange of matter between the two. The change of the mass fractions of species $i$ in the gas phase is correspondingly determined by the species production rate due to the particle-to-gas reactions, $\omega_{p g, i}$ and the gas-to-gas reactions, $\omega_{g g, i}$.

The heterogeneous particle-to-gas reactions are determined by the set of reactions listed in Table 1 . The reaction scheme is based on the turnover concept of Haynes (2001) [9], in which the carbon atoms that desorb from the carbonaceous matrix as $\mathrm{CO}$ or $\mathrm{CO}_{2}$ expose an underlying carbon atom that becomes a free carbon site $\left(\mathrm{C}_{f}\right)$, a carbon site available for adsorption of gas phase species. In the mechanism, $\mathrm{C}(\mathrm{O})$ and $\mathrm{C}(\mathrm{CO})$ represent an adsorbed oxygen atom and carbon monoxide, respectively, and $\mathrm{C}_{2}\left(\mathrm{O}_{2}\right)$ represents two adjacent carbon sites each having an adsorbed oxygen atom. Carbon dioxide $\left(\mathrm{CO}_{2}\right)$ is desorbed from this surface complex. The mechanism has been shown to accurately characterize corn stover char gasification in a fluidized bed environment, yielding predicted $\mathrm{CO}$ and $\mathrm{CO}_{2}$ concentration profiles through the bed that agree with the ones measured [10, 11]. The Arrhenius parameters for the rate coefficients shown in the table were obtained for the char of Wyodak Coal, a sub-bituminous coal from Wyoming, using data obtained in a thermogravimetric analyzer. The experimental procedure and least squares process used to determine kinetic and thermochemical parameters in the reaction mechanism are described by Tilghman and Mitchell [12]. All parameters are reasonable and supportable - all activation energies are in expected ranges. The mechanism faithfully describes measured rate phenomena over the intended ranges of temperatures, pressures and reactive gas concentrations. 
Table 1: Arrhenius parameters for the surface reactions for Wyodak coal. The unit of both the activation energy $E_{k}$ and the distribution width $\sigma_{k}$ is $\mathrm{MJ} / \mathrm{kmol}$.

\begin{tabular}{|c|c|c|c|c|c|c|}
\hline Nr. & \multicolumn{3}{|c|}{ Reaction } & $A_{k}$ & $E_{k}$ & $\sigma_{k}$ \\
\hline $\mathrm{R} 1$ & $C_{f}+C_{2}$ & $\leftrightarrow$ & $C(O)+C O$ & $3.70 \times 10^{6}$ & 161 & 0 \\
\hline $\mathrm{R} 2$ & $C_{b}+C(O)+C O_{2}$ & $\rightarrow$ & $C(O)+2 C O$ & $1.26 \times 10^{11}$ & 276 & 0 \\
\hline R3 & $C(C O)$ & $\leftrightarrow$ & $C O+C_{f}$ & $1.00 \times 10^{13}$ & 455 & 53 \\
\hline $\mathrm{R} 4$ & $\mathrm{CO}+\mathrm{C}(\mathrm{CO})$ & $\rightarrow$ & $\mathrm{CO}_{2}+2 \mathrm{C}_{f}$ & $9.80 \times 10^{9}$ & 270 & 0 \\
\hline $\mathrm{R} 5 \mathrm{a}$ & $2 C_{f}+O_{2}$ & $\rightarrow$ & $C(O)+C O$ & $5.00 \times 10^{16}$ & 150 & 0 \\
\hline $\mathrm{R} 5 \mathrm{~b}$ & $2 C_{f}+O_{2}$ & $\rightarrow$ & $\mathrm{C}_{2}\left(\mathrm{O}_{2}\right)$ & $4.00 \times 10^{13}$ & 93 & 0 \\
\hline $\mathrm{R} 6$ & $C_{f}+C(O)+O_{2}$ & $\rightarrow$ & $\mathrm{C}(\mathrm{O})+\mathrm{CO}_{2}+$ & $1.50 \times 10^{13}$ & 78 & 0 \\
\hline R7 & $b+C_{f}+C(O)+O_{2}$ & $\rightarrow$ & $2 C(O)+C O$ & $2.10 \times 10^{13}$ & 103 & 0 \\
\hline $\mathrm{R} 8$ & $C_{b}+C(O)$ & $\rightarrow$ & $C O+C_{f}$ & $1.00 \times 10^{13}$ & 353 & 28 \\
\hline $\mathrm{R} 9$ & $C_{b}+C_{2}\left(O_{2}\right)$ & $\rightarrow$ & $\mathrm{CO}_{2}+2 C_{f}$ & $1.00 \times 10^{13}$ & 304 & 33 \\
\hline
\end{tabular}

For this set of $N_{r}=9$ heterogeneous reactions, the generalized equation for surface adsorption and desorption due to reaction $k$ is

$$
\sum_{i=1}^{N_{\mathrm{s}, \mathrm{gas}}+N_{\mathrm{s}, \mathrm{ads}}} \nu_{i, k} \alpha_{i} \leftrightarrow \sum_{i=1}^{N_{\mathrm{s}, \mathrm{gas}}+N_{\mathrm{s}, \mathrm{ads}}} \nu_{i, k}^{\prime} \alpha_{i}
$$

where $N_{\mathrm{s}, \text { gas }}$ is the number of gas phase species, $N_{\mathrm{s}, \text { ads }}$ is the number of adsorbed species and $\alpha_{i}$ is a symbol representing species $i$. Furthermore the stoichiometric coefficients of the species on the reactant and product sides of the reactions are given by $\nu_{i, k}$ and $\nu_{i, k}^{\prime}$, respectively.

Rate coefficients for the reverse reactions are determined from the forward rate coefficients and the equilibrium constants, which are calculated from the heats of formation and the absolute entropies of the species involved in the reactions. The enthalpies and absolute entropies of the adsorbed species were determined in the work by Tilghman and Mitchell [12]. They show that the absolute entropy of an adsorbed species can be found by

$$
S_{\mathrm{ad}, i}=S_{\mathrm{gas}, i}\left(A_{1} T_{c}+A_{2}\right)-3.3 R
$$

when $S_{\mathrm{gas}, i}$ is the absolute entropy of the gas phase species and the coefficients $A_{1}$ and $A_{2}$ are given in Table 2. Although a slight temperature dependence 
on the heats of formation of adsorbed species was noted (more so for $\mathrm{C}(\mathrm{CO})$ ), constant values were recommended by Tilghman and Mitchell [12], as given in Table 2.

Table 2: Parameters for absolute entropy, $S_{\mathrm{ad}, i}=S_{\mathrm{gas}, i}\left(A_{1} T_{c}+A_{2}\right)-3.3 R$, together with the heat of formation for adsorbed species $i$ on Wyodak coal char.

\begin{tabular}{l|cc|c}
\hline Species & $A_{1}[1 / \mathrm{K}]$ & $A_{2}[-]$ & $\Delta H_{a d, i}[\mathrm{MJ} / \mathrm{kmol}]$ \\
\hline $\mathrm{C}(\mathrm{O})$ & 0 & 0.72 & -145 \\
$\mathrm{C}(\mathrm{CO})$ & $10^{-4}$ & 0.6 & -215 \\
\hline
\end{tabular}

\subsection{The governing equations for the gas phase}

The gas phase is described by three governing equations. The first equation determines the evolution of the mass of the gas phase, $m_{g}$, by allowing for mass to be added to the gas phase due to reactions with the solid phase. This equation is given by

$$
\frac{d m_{g}}{d t}=\frac{m_{g}}{\rho_{g}} \sum_{i=1}^{N_{\mathrm{s}, \text { gas }}} \omega_{p g, i} M_{i}
$$

where $\rho_{g}$ is the mass density of the gas phase and $M_{i}$ is the molar mass of species $i$. The second equation controls the total mass of any species $i\left(m_{g} Y_{i}\right)$ in the gas phase and is given by

$$
\rho_{g} \frac{d Y_{i}}{d t}+Y_{i} \sum_{k=1}^{N_{\mathrm{s}, \mathrm{gas}}} \omega_{p g, k} M_{k}=\left(\omega_{g g, i}+\omega_{p g, i}\right) M_{i} .
$$

where $Y_{i}$ is the mass fraction of species $i$. The energy equation, the third equation of interest, is expressed as

$$
\rho_{g} c_{p, g} \frac{d T_{g}}{d t}+\sum_{i=1}^{N_{\mathrm{s}, \mathrm{gas}}} h_{i}\left(\omega_{g g, i}+\omega_{p g, i}\right) M_{i}=n_{p}\left(Q_{h}+Q_{c}\right) .
$$

where $h_{i}$ is the enthalpy of species $i, T_{g}$ is the temperature of the gas, $c_{p, g}$ is the heat capacity of the gas mixture at constant pressure, $Q_{c}$ is the heat transfer from the particle to the gas mixture via convection and conduction 
and $Q_{h}$ is the energy transfer from the solid phase to the gas phase due to heterogeneous reactions.

The combined convective and conductive heat transfer between a particle and the gas is given by

$$
Q_{c}=H A_{p}\left(T_{p}-T_{g}\right)
$$

when $T_{p}$ is the temperature of the particle, $A_{p}$ is the outer surface of the particle of radius $r_{p}\left(A_{p}=4 \pi r_{p}^{2}\right)$ and $H$ is the heat transfer coefficient that can be expressed as $[16]$

$$
H=\frac{\mathrm{Nu} k_{g}}{2 r_{p}} \frac{B}{\exp (B)-1} .
$$

Here, $\mathrm{Nu}$ is the Nusselt number, $k_{g}$ is the thermal conductivity of the gas mixture, $B$ is the Stefan flow constant [17], given by

$$
B=\frac{\dot{m}_{p} c_{p, g}}{2 \pi r_{p} \mathrm{Nu} k_{g}}
$$

and $\dot{m}_{p}=d m_{p} / d t$.

The above expressions are strictly valid only when the inter-particle distance is much larger than the sum of the particle radius and its boundary layer thickness. This means that $\frac{4}{3} \pi\left(r_{p}+\delta_{b}\right)^{3} N_{p} \ll V$, where $\delta_{b}$ is the particle boundary layer thickness. If this is not the case, the particle boundary layers may be overlapping, and Eq. (5) is no longer valid. It is also assumed that $\delta_{b}$ is much thinner than the average distance between particles, and as such, any mass transferred from the particle to the gas immediately appears in the far field (away from the particle), and vice versa.

The enthalpy transfer between the particles and the gas due to heterogeneous reactions is

$$
Q_{h}=\sum_{i=1}^{N_{\mathrm{s}, \mathrm{gas}}} \frac{\omega_{p g, i} h_{i}\left(T^{\prime}\right) M_{i}}{n_{p}} .
$$

In the above expression, if the species $i$ is a gaseous reactant, the value of $T^{\prime}$ equals the temperature of the gas mixture while if it is a product of a heterogeneous reaction $T^{\prime}$ is the particle temperature.

The equation of state for a perfect gas is used to close the above set of equations, and is expressed as

$$
P=\frac{\rho_{g} R T_{g}}{\bar{M}}
$$


where the mean molar mass is

$$
\bar{M}=\frac{1}{\sum_{i=1}^{N_{\mathrm{s}, \text { gas }}} Y_{i} / M_{i}} .
$$

\subsection{Governing solid phase equations}

In the previous subsection, the governing equations that describe the effects of mass transport and chemical reaction in the gas phase were presented. Presented in this subsection are the governing equations that describe mass transport and chemical reaction within the solid phase, the char particle.

\subsubsection{Particle mass}

The evolution equation for the carbonaceous fraction of the char particle mass is given by

$$
\frac{d m_{c}}{d t}=\dot{m}_{c}=-S_{t} M_{c} \hat{R}_{c}
$$

where $S_{t}$ is the total surface area of the carbonaceous part of the particle, $M_{c}$ is the molar mass of carbon and $\hat{R}_{c}$ is the molar reaction rate of the carbon. Since $S_{t}=S_{g c} m_{c}$ (where $S_{g c}$ is the specific surface area of the carbonaceous part of the char particle), Eq. (11) can be rewritten as

$$
\frac{1}{m_{c}} \frac{d m_{c}}{d t}=\frac{\dot{m}_{c}}{m_{c}}=-S_{g c} \hat{R}_{c} M_{c}
$$

Given a molar reaction rate for reaction $k, \hat{R R_{k}}$, the production rate of carbon per total particle surface area is

$$
R_{c, k}=M_{c} \sum_{i=1}^{N_{\mathrm{s}, \mathrm{gas}}} \hat{R R_{k}}\left(\nu_{i, k}^{\prime}-\nu_{i, k}\right) a_{c, i}
$$

where $a_{c, i}$ is the number of carbon atoms in species $i$. In light of Eq. (12), the carbon consumption rate due solely to reaction $k$ may be written as

$$
\dot{m}_{c, k}=-S_{t} R_{c, k}
$$

The formula used for the specific surface area of the carbonaceous part of a porous char particle was based on the work of Bhatia and Perlmutter [18] who derived the following expression for the volume specific surface area 
$\left(S_{v c}\right)$ evolution during char conversion under kinetically controlled gasification conditions (i.e., for gasification in the zone I conversion regime)

$$
S_{v c}=S_{v c, 0}(1-x) \sqrt{1-\psi \ln (1-x)} .
$$

Here, $\psi$ is the structural parameter and $x$ is the carbon conversion. In the zone I conversion regime, the apparent density of the carbonaceous part of the particle varies proportionally with the carbonaceous mass of the particle, and as such $m_{c} / m_{c, 0}=\rho_{c} / \rho_{c, 0}=1-x$. Without any loss of generality, the volume specific surface area can be rewritten as

$$
S_{v c}=S_{v c, 0} \rho_{c} / \rho_{c, 0} \sqrt{1-\psi \ln \left(\rho_{c} / \rho_{c, 0}\right)} .
$$

Since the mass specific surface area $S_{g c}$ equals $S_{v c} / \rho_{c}$, the following expression can be used to follow the mass specific surface area evolution during char conversion:

$$
S_{g c}=S_{g c, 0} \sqrt{1-\psi \ln \left(\frac{\rho_{c}}{\rho_{c, 0}}\right)} .
$$

Note that this expression is valid not only for the char gasification in the zone I conversion regime but also for gasification in the zone III regime. In the zone III conversion regime, reaction is confined to the particle periphery and the apparent density of the particle remains constant; the mass specific area should remain constant as well, as dictated by Eq. (17).

Since Eq. (17) holds both for gasification in the zone I and III conversion regimes, it seems reasonable to use the equation to determine mass specific surface area in the zone II conversion regime, as was done in the approach of Mitchell et al. [14]. In the zone II conversion regime, due to gasification on the internal and external particle surfaces both apparent density and particle size are reduced with mass loss. In this approach, the mass specific surface area will vary as a result of changes in apparent density. As described below, a mode of conversion sub-model is used to govern the variations in particle size and apparent density with mass loss. It should be noted that although there are gradients in apparent density inside the particle, the mass specific surface area is taken to be uniform. The impacts of this assumption are inconsequential.

In the above, focus has been on the carbonaceous part of the char particle. In order to find the total mass of the char particle, account must be made also for the ash content of the particle. It is assumed that any mineral 
matter in the char is non-reactive and remains with the particle during the course of char conversion. Furthermore, it is assumed that any ash liberated on the particle surface is instantaneously redistributed throughout the particle. For some biomass materials, this assumption may be inadequate since some biomass ashes have relatively low fusion temperatures and may even be volatile. At sufficiently high temperatures the ash, in particular biomass ash, may not be truly non-reactive nor non-catalytic. Including reactive and catalytic effects of the ash is, however, beyond the scope of this work.

With these assumptions, the mass of the ash-containing char particle $\left(m_{p}\right)$ is given by

$$
m_{p}=m_{c}+m_{a}=m_{c}+X_{a} m_{p, 0}=m_{c}+\frac{X_{a} m_{c, 0}}{1-X_{a}}
$$

where $m_{a}$ is the mass of the mineral matter and $X_{a}$ is the initial mass fraction of ash in the char particle. It is assumed that the particle's total specific surface area, $S_{g p}$, is distributed between the ash and carbonaceous components on a mass-weighted basis. Therefore,

$$
S_{g p}=\left(1-x_{a}\right) S_{g c}+x_{a} S_{g a},
$$

where $S_{g a}$ is the specific surface area of the ash (taken to be $5 \mathrm{~m}^{2} / \mathrm{g}$ for both the coal and biomass ashes), $x_{a}$ is the instantaneous mass fraction of the ash in the particle, and $S_{g c}$ is the specific surface area of the carbonaceous particle material, which varies with conversion as described by Bhatia and Perlmutter.

\subsubsection{Particle temperature}

The char particle temperature equation is governed by the following energy balance relationship:

$$
\frac{d T_{p}}{d t}=\frac{1}{m_{p} c_{p, p}}\left(Q_{\mathrm{reac}}-Q_{c}+Q_{\mathrm{rad}}\right) .
$$

Here, $c_{p, p}$ is the specific heat capacity of particle and $Q_{\text {reac }}, Q_{c}$ and $Q_{\mathrm{rad}}$ are the heating due to heterogeneous reactions, convective and conductive transport from the particle to the gas mixture and radiation, respectively. The net heat of reaction of the particle due to solid phase reactions is

$$
Q_{\text {reac }}=S_{t} \sum_{k=1}^{N_{r}} R \hat{R}_{k} \hat{q}_{\text {reac }, k}
$$


where $N_{r}$ is the number of heterogeneous reactions and $q_{\text {reac, } k}$ is the heat of reaction of surface reaction $k$. The molar heat of reaction for reaction $k$ is given by

$$
\hat{q}_{\mathrm{reac}, k}=\sum_{i=1}^{N_{\mathrm{s}, \mathrm{gas}}+N_{\mathrm{s}, \text { ads }}}\left(\nu_{i, k}-\nu_{i, k}^{\prime}\right) h_{i}
$$

where $h_{i}$ is the enthalpy of formation of species $i$, evaluated at the particle temperature.

The radiant energy flow from the particle to the wall is described in the traditional fashion by the relationship

$$
Q_{\mathrm{rad}}=\epsilon \sigma A_{p}\left(T_{w}^{4}-T_{p}^{4}\right)
$$

where $\epsilon, \sigma$ and $T_{w}$ are the emissivity, the Stefan-Boltzmann constant and the wall temperature, respectively. In this traditional approach, gas phase radiation and radiation between particles have been neglected. In our approach, account is made for inter-particle radiation, in which the radiative loss from the particle depends on its position in the particle cloud, whether at the cloud periphery, at its center or somewhere in between. We define the average particle, which is positioned somewhere in between, such that $Q_{\mathrm{rad}} n_{p} V=E_{\text {wall,net }}$, where $E_{\text {wall,net }}$ is the net radiation transferred to the wall. By construct, it is now ensured that the heat load on the walls is that which would be experienced from a particle cloud consisting of particles having the same properties as the particle considered. It is also ensured that the cooling of the particle considered equals the cooling that the average particle in a particle cloud would experience.

When accounting for inter-particle radiation, and picking the average particle within the enclosure, the radiative term $\left(Q_{\mathrm{rad}}\right)$ can be expressed as $[22]$

$$
Q_{\mathrm{rad}}=\frac{3 g(\beta) \sigma}{n_{p} R}\left(T_{w}^{4}-T_{p}^{4}\right)
$$

where

$$
g(\beta)=1-\frac{1}{2 \beta^{2}}+e^{-2 \beta}\left(\frac{1}{\beta}+\frac{1}{2 \beta^{2}}\right) .
$$

In this expression, $\beta$ is the optical depth $(\beta=a R)$, where $R$ is the radius of the enclosure and $a$ is the absorption coefficient of the cloud due to the absorption of radiant energy by the particles. Inter-particle radiation can be neglected when the optical depth is much smaller than unity, i.e. when 
$\beta=a R \ll 1$. However, this is not the case in typical gasification reactors: inter-particle radiation should be taken into account when modeling the char gasification process [22].

\subsubsection{Adsorbed species}

The number of moles of species $j$ adsorbed on the particle surface is given by

$$
N_{j}=C_{s, j} S_{t}
$$

where $C_{s, j}$ is the concentration of adsorbed species $j$ on the carbonaceous surface. Differentiating this equation with respect to time and rearranging yields the following expression for the rate of change in the concentration of the adsorbed species

$$
\frac{d C_{s, j}}{d t}=\frac{1}{S_{t}} \frac{d N_{j}}{d t}-\frac{C_{s, j}}{S_{t}} \frac{d S_{t}}{d t} .
$$

The rate of change of moles of adsorbed species $j$ depends on the rates of the reactions in which it is involved, and is given by

$$
\frac{d N_{j}}{d t}=\hat{R}_{j} S_{t}
$$

where $\hat{R}_{j}$ is the molar production rate of adsorbed species $j$ (see Eq. (45)) and $S_{t}$ is the total surface area. Employing Eq. (28) in Eq. (27) reveals that

$$
\frac{d C_{s, j}}{d t}=\hat{R}_{j}-\frac{C_{s, j}}{S_{t}} \frac{d S_{t}}{d t}
$$

It is convenient to recast the above equations in terms of the adsorbed species site fractions instead of concentrations. The site fraction of species $j$ is defined as follows:

$$
\Theta_{j}=\frac{C_{s, j}}{\xi_{n}},
$$

where $\xi_{n}$ is the total surface concentration of all carbon sites, whether occupied or not. This implies that

$$
\sum_{j=1}^{N_{\mathrm{s}, \text { ads }}} \Theta_{j}=1
$$


where $N_{\text {s,ads }}$ is the total number of adsorbed species. Note that free carbon sites are counted as a species. Employing the definition of the site fractions yields the following equation for the rate of change in the site fraction for adsorbed species $j$ (except for the free carbon sites):

$$
\frac{d \Theta_{j}}{d t}=\frac{\hat{R}_{j}}{\xi_{n}}-\frac{\Theta_{j}}{S_{t}} \frac{d S_{t}}{d t} .
$$

By differentiating Eq. (17) and employing Eqs. (11) and (12) together with the relation between conversion and the mass of the char particle: $x=$ $1-m_{c} / m_{c, 0}$, the evolution of the total surface area can be expressed as follows:

$$
\frac{1}{S_{t}} \frac{d S_{t}}{d t}=\left(\frac{S_{t, 0}^{2} \psi(1-x)^{2}}{2 S_{t}^{2}}-1\right) S_{g c} M_{c} \hat{R}_{c}=-A \hat{R}_{c}
$$

where

$$
A=\left(1-\frac{S_{t, 0}^{2} \psi(1-x)^{2}}{2 S_{t}^{2}}\right) S_{g c} M_{c} .
$$

With this, the rate of change in the site fraction of adsorbed species $j$ (Eq. (32)) can be written as

$$
\frac{d \Theta_{j}}{d t}=\frac{\hat{R}_{j}}{\xi_{n}}+A \hat{R}_{c} \Theta_{j} .
$$

Since the site fraction must sum to unity, the fraction of free carbon sites is determined from

$$
\Theta_{c f}=1-\sum_{i \neq c f} \Theta_{i}
$$

\subsection{Species concentrations at the particle surface}

Since the model solves for the species mole fraction of the gas phase, the mole fractions of the reactants in the ambient, $X_{i, \infty}$, are known at any time. The reactant mole fractions at the particle surface, $X_{i, s}$, are controlled by the transport from the ambient through the boundary layer to the external surface of the particle. In steady state, the equation balancing the flux of species $i$ through the boundary layer with its net production due to heterogeneous reactions is given by

$$
\dot{n}_{i}-X_{i, s} \dot{n}_{\text {total }}=-k_{i m}\left(X_{i, \infty}-X_{i, s}\right) .
$$


where $k_{i m}$ is the mass transfer coefficient of species $i$. The second term on the left hand side of Eq. (37) is due to the Stefan flow. The molar flux of each gaseous species $i$ at the external particle surface can be expressed as

$$
\dot{n}_{i}=\sum_{k=1}^{N_{r}} \dot{n}_{i, k}
$$

where the molar flux of species $i$ due to heterogeneous reaction $k, \dot{n}_{i, k}$, is given by

$$
\dot{n}_{i, k}=\left(\nu_{i, k}^{\prime}-\nu_{i, k}\right) \hat{R R_{k}} \frac{S_{t}}{A_{p}} .
$$

Knowing the molar flux of species $i$ at the external surface of the particle, $\dot{n}_{i}$, the rate of production of species $i$ from a single particle is given by $\dot{n}_{i} A_{p}$ since $\dot{n}_{i}$ is in units of the external particle surface area. In order to obtain the species production rate due to heterogeneous reactions in all the particles, $\omega_{p g, i}$, which appears as a source term in the governing gas phase species equation (Eq. (3)), the entire cloud of particles must be taken into account. This is done by multiplying the rate of production of species $i$ per particle $\left(\dot{n}_{i} A_{p}\right)$ with the particle number density $n_{p}$. The production rate of species $i$ due to surface reactions is then

$$
\omega_{p g, i}=\dot{n}_{i} A_{p} n_{p}
$$

When the inter-particle distance is much larger than the particle radius, the mass transfer coefficient, $k_{i m}$, is determined from the Sherwood number, assumed to be 2 for the low Reynolds number flows of interest. As such

$$
k_{i m}=\frac{C_{g} D_{i m} \mathrm{Sh}}{2 r_{p}}=\frac{C_{g} D_{i m}}{r_{p}}
$$

where $D_{i m}$ is the molecular bulk diffusivity of species $i$ and the total gas concentration in the thin film surrounding the particle, $C_{g}$, is

$$
C_{g}=\frac{\rho}{\bar{M}}=\frac{P}{R T_{f}}
$$

In the above equation, $\bar{M}$ is the average molecular weight of the bulk gas, while $T_{f}$ is the characteristic film temperature, which is given by:

$$
T_{f}=T_{p}+a\left(T_{g}-T_{p}\right)
$$


when $a=1 / 3$.

Knowing that $\dot{n}_{\text {total }}=\sum_{i} \dot{n}_{i}$ the Newton-Raphson method can be employed to find values of $X_{i, s}$ that satisfy Eq. (37). For infinitely fast mass diffusion this equation simplifies to $X_{i, s}=X_{i, \infty}$.

\subsection{Surface reactions}

The rate of reaction $k$ is expressed in terms of the concentrations of the species involved in the reaction via the following relation:

$$
\hat{R R_{k}}=k_{k} \prod_{i=1}^{N_{\mathrm{s}, \text { gas }}+N_{\mathrm{s}, \mathrm{ads}}} C_{i}^{\nu_{i, k}}
$$

when $k_{k}$ is the rate coefficient of reaction $k$ and $C_{i}$ is the concentration of species $i$. The molar production rate of species $i$ due to all reactions is

$$
\hat{R}_{i}=\sum_{k=1}^{N_{r}}\left(\nu_{i, k}^{\prime}-\nu_{i, k}\right) \hat{R R_{k}}
$$

The temperature dependence of the reaction rate coefficient for each reaction is expressed in Arrhenius form:

$$
k_{k}=A_{k} \exp \left(-E_{k} /\left(R T_{p}\right)\right) .
$$

In order to account for the variations in the strengths of adsorbed species, the rate of the desorption reactions are modeled using a distributed activation energy approach (see for example Ma et al. [23]). The distribution in

activation energy, $f(E)$, is assumed to be a Gaussian, with $E_{k}$ and $\sigma_{k}$ as the mean activation energy and the standard deviation for reaction $k$, respectively. When allowance is made for a distribution of activation energies, the reaction rate coefficient for the reaction is given by

$$
k_{k}=\int_{0}^{\infty} k_{k}(E) f(E) d E
$$

where the activation energy distribution is expressed as

$$
f(E)=\frac{1}{\sigma \sqrt{2 \pi}} \exp \left[-\frac{1}{2}\left(\frac{E-E_{k}}{\sigma}\right)^{2}\right] .
$$


With this treatment of distributed activation energies, it is implied that desorption and adsorption of oxide complexes have similar activation energy distributions and consequently, oxide complexes of a given activation energy are replenished at nearly the same rate as they are exhausted rendering a nearly unchanged activation energy distribution. In effect, we assume $f(E)$ to not vary in time. This assumption has been verified by Du et. al. [24].

The rates of the desorption reactions $\mathrm{R} 3, \mathrm{R} 8$ and $\mathrm{R} 9$ in the reaction mechanism shown in Table 1 are described by distributions of activation energies. The values for $E_{k}$ and $\sigma_{k}$ for each reaction were determined by measuring char desorption rates in temperature-programmed desorption (TPD) experiments, as described by Tilghman and Mitchell [12]

\subsection{Internal particle burning and the effectiveness factor}

Allowances are made for both bulk and Knudsen diffusion in the pores of the char particle. The Knudsen diffusion coefficient for species $i$ is given by

$$
D_{\mathrm{K}, i}=\frac{2 r_{\text {pore }} \theta}{3 \tau} \sqrt{\frac{8 R T_{p}}{\pi M_{i}}}
$$

where $\tau$ is the tortuosity factor (introduced in order to account for the fact that the pores are randomly oriented and not purely in the radial direction) and $r_{\text {pore }}$ is the mean pore radius, estimated from the particle volume $\left(V_{p}\right)$, porosity $(\theta)$ and roughness factor $\left(f_{r}\right)$ in the following way: $r_{\text {pore }}=2 f_{r} \theta V_{p} / S_{t}$. The effective diffusion coefficient of species $i$ is given by

$$
\frac{1}{D_{\mathrm{eff}, i}}=\frac{1}{D_{i}}+\frac{1}{D_{\mathrm{K}, i}}
$$

As pore size decreases, Knudsen diffusion become more important than bulk diffusion and the diffusion coefficient decreases, while approaching $D_{K, i}$.

If mass transport rates within the particle are slow compared to the heterogeneous reaction rates, the reactant concentration inside the particle will not be uniform, and the overall particle reaction rate will be lower than it would be if the reactant concentration were uniform. Taking the approach of Thiele [20] to account for the reduced reaction rate when there is a gradient in the reactive gas concentration inside the particle, the effectiveness factor for reactant gas $i\left(\eta_{i}\right)$ is defined as:

$$
\eta_{i}=\frac{\text { Actual overall reaction rate of reactant species } i}{\text { Maximum possible reaction rate of reactant species } i}=\frac{\hat{R}_{i}}{\hat{R}_{i, \max }} \text {. }
$$


The effectiveness factor is related to the Thiele modulus, $\phi_{i}$, a dimensionless parameter that is a relative measure of chemical reaction rates to diffusion rates inside the particle. For a single, irreversible first-order reaction between gas phase species $i$ and a porous spherical particle, Thiele showed that

$$
\eta_{i}=\frac{3}{\phi_{i}}\left[\frac{1}{\tanh \left(\phi_{i}\right)}-\frac{1}{\phi_{i}}\right]
$$

where the Thiele modulus is defined as

$$
\phi_{i}=r_{p} \sqrt{\frac{\hat{R}_{i} \rho_{p} S_{g c}}{C_{g} X_{i} D_{\mathrm{eff}, \mathrm{i}}}} .
$$

In previous work employing $\mathrm{O}_{2}$ as the reactive gas, Mitchell et al. (2007) [14] showed that the reaction mechanism in Table 1 yields a $\phi_{\mathrm{O} 2}-\eta_{\mathrm{O} 2}$ relationship that nearly follows the first order results of Thiele. When $\mathrm{CO}_{2}$ is the reactive gas, we also assume a $\phi_{\mathrm{CO} 2}-\eta_{\mathrm{CO} 2}$ relationship consistent with first order kinetics. Direct numerical simulations of a spherical char particle exposed to various gaseous environments at temperatures up to $2200 \mathrm{~K}$ were performed in a manner similar to that undertaken in previous work by Mitchell et al. (2007) [14], but using the reaction mechanism presented in Table 1 (see Mitchell (2014) [15]). The results support the use of the $\phi_{C O 2}-\eta_{C O 2}$ relationship for irreversible, first-order kinetics.

In terms of an effectiveness factor, the reaction rate for reaction $k$, as given by Eq. (44), is augmented to read

$$
\hat{R R_{k}}=\eta_{k} k_{k} \prod_{i=1}^{N_{\mathrm{s}, \mathrm{gas}}+N_{\mathrm{s}, \mathrm{ads}}} C_{i}^{\nu_{i, k}}
$$

where $\eta_{k}$ is the effectiveness factor of reaction $k$, which equals the effectiveness factor for the gas phase reactant of reaction $k$. If reaction $k$ has no gas phase reactant the effectiveness factor for reaction $k$ is set to one. Likewise, if there is a net production of the reactant species in reaction $k$ the effectiveness factor of reaction $k$ is also set to one.

\subsection{Mode of char particle conversion}

The relation between char particle radius, apparent density and mass is defined by the mode of conversion. In the zone I conversion regime, the particle consumption is uniform such that the particle radius is constant, while 
in the zone III conversion regime the apparent density of the particle is constant. For the intermediate case, i.e. in the zone II conversion regime, both particle size and apparent density decrease with conversion. Haugen, Tilghman and Mitchell [21] showed analytically that when the apparent density in a thin layer at the periphery of the char particle falls to zero (i.e. when the mass in the layer is completely consumed), the particle diameter starts to decrease. The time at which this occurs, $\tau_{c}$, is determined from the following relationship

$$
\rho_{c, 0}=\int_{0}^{\tau_{c}} R\left(r_{p}, t^{\prime}\right) d t^{\prime}
$$

where $\rho_{c, 0}$ is the initial apparent density of the carbonaceous part of the char particle and $R\left(r_{p}, t\right)$ is the overall char conversion rate at the external surface of the particle of radius $r_{p}$ at time $t$. For $t>\tau_{c}$, when the particle radius is decreasing, the model developed by Haugen et al. [21] takes the approach of Thiele when describing the effects of non-uniform concentration gradients inside the particle. The authors derived the following expression for the rate of change of radius and apparent density during char conversion

$$
\text { for } t \leq \tau_{c}
$$

$$
\frac{d r_{p}}{d t}=0 \quad \text { and } \quad \frac{d \rho_{p}}{d t}=\frac{d m_{c}}{d t} \frac{1}{V_{p}}
$$

and for $t>\tau_{c}$

$$
\frac{d r_{p}}{d t}=\frac{d m_{c}}{d t} \frac{1-\eta}{4 \pi r_{p}^{2} \rho_{p}} \text { and } \quad \frac{d \rho_{p}}{d t}=\frac{d m_{c}}{d t} \frac{\eta}{V_{p}}
$$

As noted, for $t<\tau_{c}$ the radius is constant, and the mass loss is due to a reduction in apparent density. For $t>\tau_{c}$, both the changes in apparent density and particle radius depend on the mass loss rate as well as on the effectiveness factor.

In contrast to the species specific effectiveness factor given by Eq. (51), the mean effectiveness factor used in Eq. (56) is defined as a mean over all reactant species such that

$$
\eta=\frac{\text { Actual overall reaction rate of all reactant species }}{\text { Maximum possible reaction rate of all reactant species }}=\frac{\hat{R}}{\hat{R}_{\max }},
$$

where

$$
\hat{R}=\sum_{i=1}^{N_{\text {reactants }}} \hat{R}_{i}
$$


and

$$
\hat{R}_{\max }=\sum_{i=1}^{N_{\text {reactants }}} \hat{R}_{i, \max }
$$

such that by employing Eq. (51) it is found that

$$
\eta=\frac{\sum_{i=1}^{N_{\text {reactants }}} \eta_{i} \hat{R}_{i, \max }}{\sum_{i=1}^{N_{\text {reactants }}} \hat{R}_{i, \max }} .
$$

In the above expression, $N_{\text {reactants }}$ represents the number of reactants of the global set of reactions. This means that a given specie may be a reactant of an individual reaction but is nevertheless not included in $N_{\text {reactants }}$ if it is not also a reactant of the global set of heterogeneous reactions. For example, for the set of reactions listed in Table $1 \mathrm{CO}$ is a reactant for several reactions, but for the global set of reactions it is a product and not a reactant, i.e. it is not counted in $N_{\text {reactants }}$.

\section{Results}

In this section, dry gasification of char in an atmosphere of 24 bar and an initial temperature of $1640 \mathrm{~K}$ is studied. The base case (Case A in Table 3) is designed to be an auto thermal mixture of oxygen and carbon dioxide, i.e. there is just enough oxygen to fuel the gasification. For particle masses $\left(7 \times 10^{-10} \mathrm{~kg}\right)$ and number densities $\left(10^{9} \mathrm{~m}^{-3}\right)$ typical for entrained flow gasifiers, the composition of the initial mixture then becomes $84 \% \mathrm{CO}_{2}$ and $16 \% \mathrm{O}_{2}$. In order to emphasize the effect of the particle number density, two different particle-to-gas mass ratios (0.18 and 0.12 ) and two different equivalence ratios (2 and 3$)$ are studied. Here, the equivalence ratio is defined as $\phi_{e q}=\left(\right.$ fuel $\left./ \mathrm{O}_{2}\right) /\left(\text { fuel } / \mathrm{O}_{2}\right)_{\text {stoich }}$. Case A represents the situation where the particle-to-gas mass ratio is 0.18 and the corresponding equivalence ratio is 3 , while cases $\mathrm{B}$ and $\mathrm{C}$ have a lower particle-to-gas mass ratio of 0.12 . In case $\mathrm{B}$, the initial gas composition is the same as in case A, yielding a lower equivalence ratio of 2 while in case $\mathrm{C}$, the equivalence ratio is kept equal to case A by substituting a portion of the oxygen $\left(\mathrm{O}_{2}\right)$ in the gas phase with nitrogen $\left(\mathrm{N}_{2}\right)$. See Tables 3 and 4 for more information on the simulations.

In Fig. 1 the conversion of the char as a function of time is shown. It can be seen that for cases $\mathrm{A}$ and $\mathrm{C}$ (with the larger equivalence ratios), it takes longer to reach full conversion of the char than for case B. It is also noted that case $\mathrm{B}$ exhibits the faster conversion rate, a consequence of both temperature 
Table 3: Physical properties for all simulations presented.

\begin{tabular}{llcl}
\hline Property & Symbol & Value & Unit \\
\hline Surf. conc. of C sites & $\xi$ & $1.08 \times 10^{-7}$ & $\mathrm{kmol} / \mathrm{m}^{2}$ \\
Spec. init. part. surf. area & $S_{g c, 0}$ & $475 \times 10^{3}$ & $\mathrm{~m}^{2} / \mathrm{kg}$ \\
Structural parameter & $\psi$ & 8 & - \\
App. density of part. & $\rho_{p}$ & 600 & $\mathrm{~kg} / \mathrm{m}^{3}$ \\
Initial particle radius & $r_{p}$ & $6.5 \times 10^{-5}$ & $\mathrm{~m}$ \\
Tortuosity factor & $\tau$ & 3 & - \\
Roughness factor & $f_{r}$ & 2 & - \\
True density of carbon & $\rho_{c, t}$ & 1800 & $\mathrm{~kg} / \mathrm{m}^{3}$ \\
\hline
\end{tabular}

Table 4: Some case specific properties.

\begin{tabular}{lllccc}
\hline Property & & Units & Case A & Case B & Case C \\
\hline Carbon to gas mass ratio & $m_{p} / m_{g}$ & {$[-]$} & 0.18 & 0.12 & 0.12 \\
Particle number density & $n_{p}$ & {$\left[10^{9} / \mathrm{m}^{3}\right]$} & 2.0 & 1.3 & 1.3 \\
Mass fraction of $\mathrm{O}_{2}$ & $Y_{\mathrm{O}_{2}}$ & {$[\mathrm{~kg} / \mathrm{kg}]$} & 0.16 & 0.16 & 0.11 \\
Mass fraction of $\mathrm{CO}_{2}$ & $Y_{\mathrm{CO}_{2}}$ & {$[\mathrm{~kg} / \mathrm{kg}]$} & 0.84 & 0.84 & 0.84 \\
Mass fraction of $\mathrm{N}_{2}$ & $Y_{\mathrm{N}_{2}}$ & {$[\mathrm{~kg} / \mathrm{kg}]$} & 0 & 0 & 0.05 \\
Equivalence ratio & $\phi_{e q}$ & {$[-]$} & 3 & 2 & 3 \\
\hline
\end{tabular}

and $\mathrm{CO}_{2}$ concentration, as can be seen in Fig. 2. It is evidenced that in case $\mathrm{C}$, peak particle and gas temperatures are lower. This is because, for fuel rich conditions, the peak temperature is determined by the amount of oxygen available, which is lower for case $\mathrm{C}$. The lesser the amount of $\mathrm{O}_{2}$, the less char is oxidized and hence the lower the peak temperature. After the oxygen has been consumed (after less then 0.1 seconds), char gasification by $\mathrm{CO}_{2}$ takes over as the dominating conversion mechanism. Due to the endothermicity of the gasification reaction this results in a temperature decrease. Char conversion due to $\mathrm{CO}_{2}$ gasification is initially slower for case $\mathrm{C}$ than for case A due to the lower temperatures of case $\mathrm{C}$, but since there are fewer particles in case $\mathrm{C}$ neither the temperature nor the $\mathrm{CO}_{2}$ concentration decrease as rapidly as for case $\mathrm{A}$. This eventually yields the higher conversion rate for case C, which is seen in Fig. 1 for $t>0.2$.

Another effect that slows down the rate of conversion of case $\mathrm{A}$ is the 


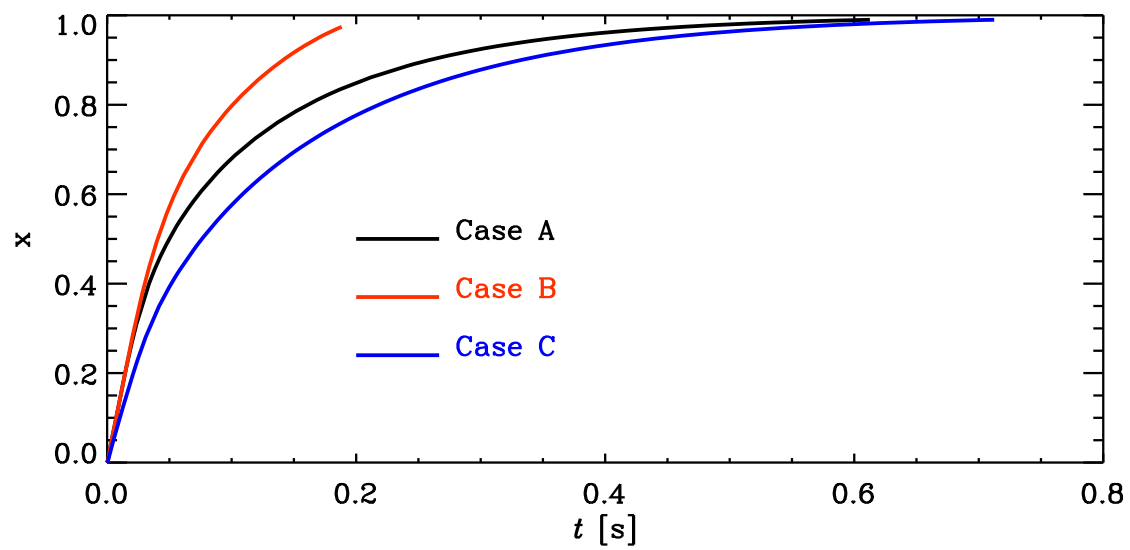

Figure 1: Carbon conversion as a function of time. (See Table 4 for descriptions of Cases A, B and C.)

buildup of large amounts of carbon monoxide in the gas (as noted in the right side of Fig. 2), which has an inhibiting effect on char gasification. From the same figure it can also be seen that the final CO mass fractions at the end of char conversion varies from 0.25 of case $\mathrm{B}$, to 0.33 of case $\mathrm{C}$ and finally to 0.46 for case A. Note that increasing the mass fraction of $\mathrm{N}_{2}$ from zero to $5 \%$, at the expense of $\mathrm{O}_{2}$, significantly changes the amount of $\mathrm{CO}$ produced.

Despite the similarity in temperature evolution for cases A and B up to peak temperatures, both the temperature and the $\mathrm{CO}_{2}$ concentrations are higher for case B after the oxygen has been consumed. This yields a much shorter time to full conversion than for both cases $\mathrm{A}$ and $\mathrm{C}$ (as noted in Fig. 1).

Both the different temperatures and species concentrations between cases $\mathrm{A}$ and $\mathrm{B}$ are directly due to the denser cloud of char particles in case A, and clearly show the effect of increasing the particle-to-gas mass ratio while keeping the gas composition constant. In the example shown here, decreasing the number of particles by $33 \%$ leads to a decrease in char conversion time by more than a factor of 3 . Calculations made keeping the equivalence ratio constant while decreasing the char particle number density, as is done in case $\mathrm{C}$, indicate that the total time to full conversion may not be very different even if the temperature and species evolutions are different.

As the char particles are consumed, the apparent density and radii of the particles will decrease. Mitchell et al. (2007) [14] and Haugen et al. (2014) 

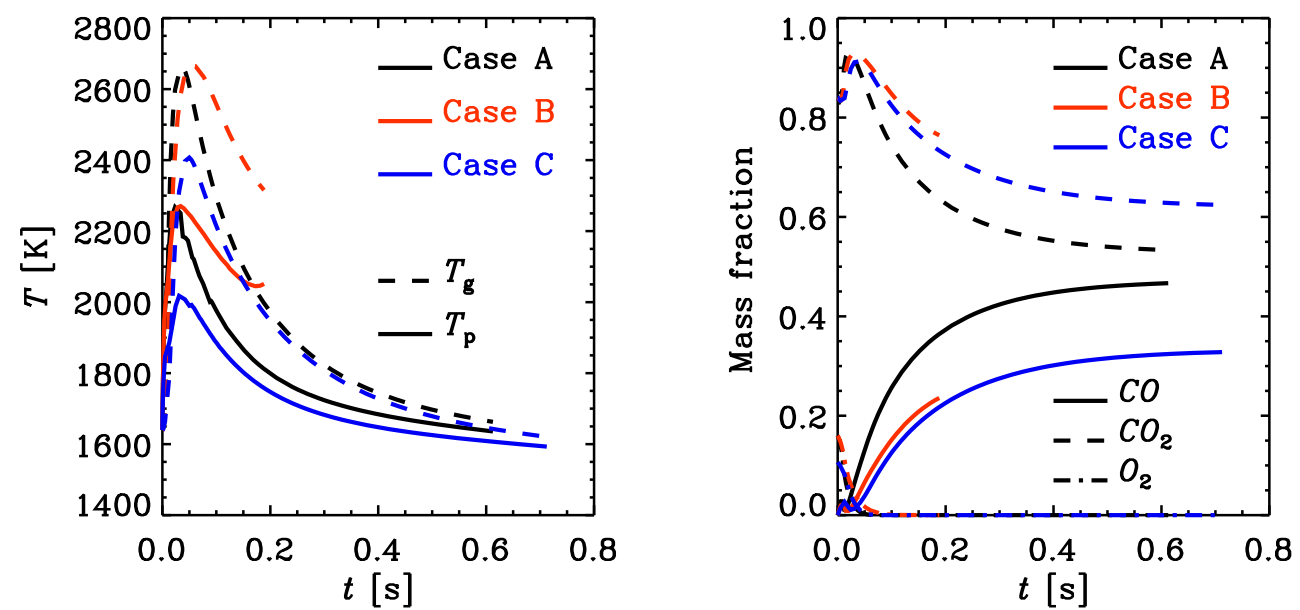

Figure 2: Particle and gas phase temperatures (left panel) and species mass fractions (right panel) as a function of time.
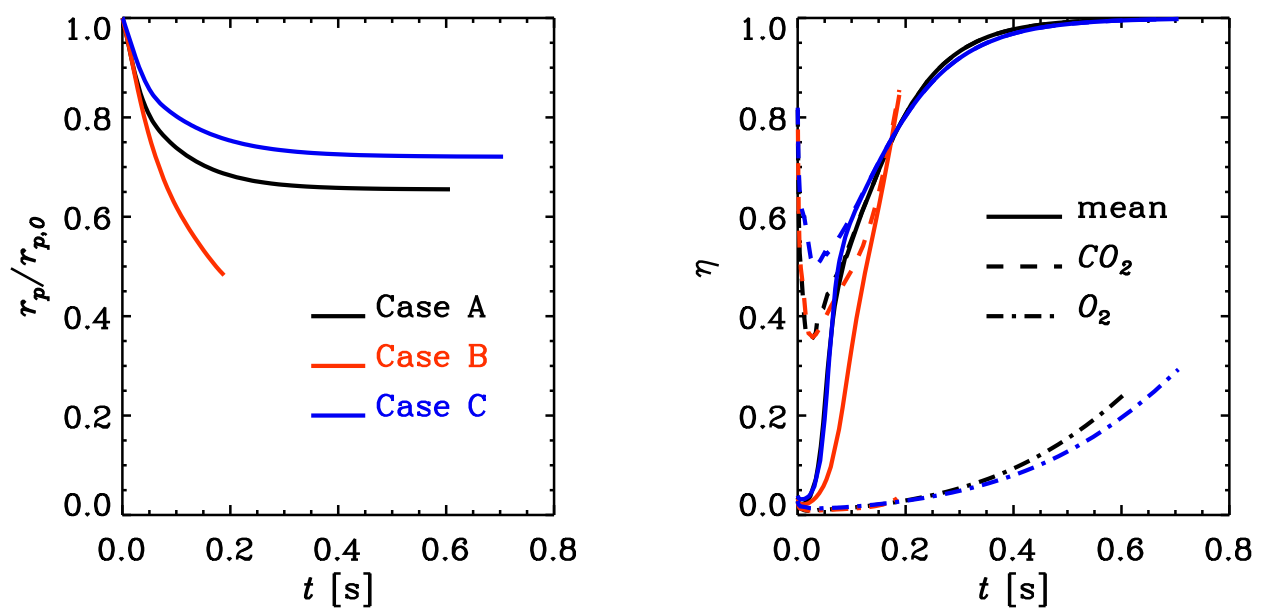

Figure 3: Particle radius (left panel) and effectiveness factors (right panel) as a function of time. In the right panel the line label "mean" correspond to the effectiveness factor as found by Eq. (57) while the species specific effectiveness factors are found from Eq. (51).

[21] showed that for a porous particle, which initially has a uniform density distribution, the particle radius will not start to decrease immediately. Haugen et al. (2014) [21] also showed that the particle radius starts to decrease 
when the char conversion roughly equals the effectiveness factor. This means that for the very small initial effectiveness factors experienced by the cases presented in this paper, the particle radius should start to decrease almost immediately. By inspecting the left plot of Fig. 3, it can be seen that this is indeed the case.

It is customary to divide char conversion into three zones. For zone I conversion the char is fully penetrated by the reactant gases and the effectiveness factor, $\eta$, is close to unity. For zone III, char conversion is diffusion controlled and all reactions are confined to the outer periphery of the particle. This corresponds to a quite small effectiveness factor. Zone II conversion is then defined as everything between zone I and III. The solid lines of the right panel of Fig. 3 shows that the effectiveness factor initially starts out being close to zero for all cases, making the reactions diffusion controlled, but as time progress (and temperatures and particle radius decrease), the mean value of the effectiveness factor increases, and eventually ends up near unity for cases $\mathrm{A}$ and $\mathrm{C}$, while it stops at a value near 0.85 for case B. From the above it can be concluded that all three cases experience zone III conversion during the initial stages of conversion, when $\eta$ is close to zero. After the initial period of conversion under zone III conditions, for all three cases the conversion rates fall into the zone II regime, where the particles are partially penetrated by the reactants. For case B, full conversion is reached under zone II conditions while for cases $\mathrm{A}$ and $\mathrm{C}$, char conversion rates fall into the zone I regime before all the carbon is consumed.

It is noted that the particle radius at the time when full conversion is reached, defined as when $x>0.99$, is significantly smaller for case $\mathrm{B}$ than for cases $\mathrm{A}$ and $\mathrm{C}$. This is due to the fact that the term $(1-\eta)$, which from Eq. (56) is seen to impact the rate of change in particle radius, is larger for case $\mathrm{B}$ when averaged over the time to full conversion.

By inspecting Fig. 4 it can be seen that the fraction of carbon sites being free $\left(\mathrm{C}_{f}\right)$ is always close to unity, indicating that the char conversion rate is adsorption limited. Having realized this, it becomes apparent that CO-inhibition is not due to adsorbed CO molecules occupying all the available sites on the char surface. Instead, the CO inhibition is caused by the increased rate of the reverse of reaction $\mathrm{R} 1$ for cases with high $\mathrm{CO}$ concentration.

For each of the cases considered, the dominant char conversion pathway is via $\mathrm{C}(\mathrm{O})$ desorption. In contrast to adsorbed $\mathrm{O}$, adsorbed $\mathrm{CO}$ is not capable of removing a carbon atom from the char surface - an adsorbed CO 


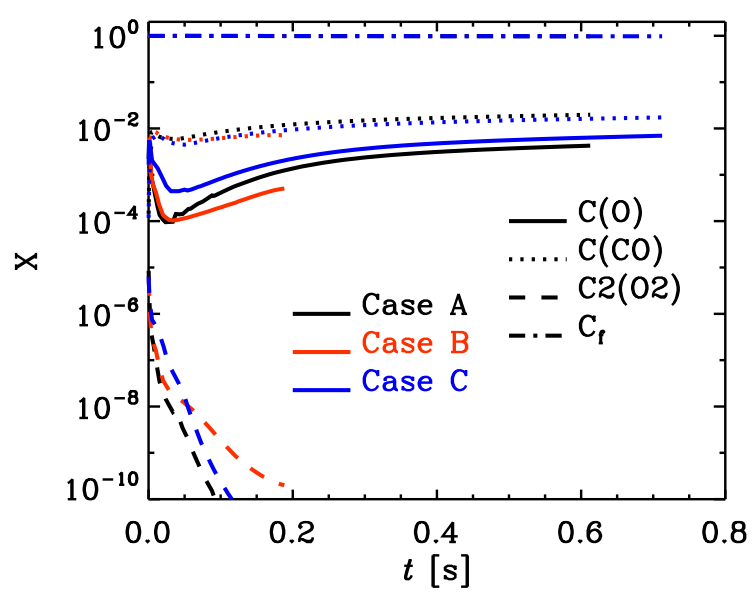

Figure 4: Surface fraction of adsorbed species for cases A and B.

molecule does not contribute to the overall char conversion rate. The $\mathrm{C}(\mathrm{O})$ concentration is, as can be seen from Fig. 4, quite low, while the concentration of adsorbed $\mathrm{CO}$ is somewhat higher, but is still found to occupy only roughly $1 \%$ of the free sites. This is due to the higher desorption rate of $\mathrm{C}(\mathrm{O})$.

In the left panel of Fig. 5, the radiative cooling of the particles is shown as a function of time for different particle cloud sizes $(R)$ and for different treatments of the radiation. The black, red and blue solid lines correspond to cases A, B and C, respectively, where the radius of the particle cloud is set to $R=1 \mathrm{~m}$. The particle cooling of case $\mathrm{B}$ is found to be stronger than for case A. This is primarily due to the lower particle number density for case B, which yields more cooling to the walls per particle. In addition to this, the cooling of case B is also increased due to the higher particle temperatures experienced in this case. For late times (after the particle temperatures have peaked) case $\mathrm{C}$ also yields slightly stronger cooling than case $\mathrm{A}$ due to the lower particle number density in case C. Before particle temperatures have peaked, however, the difference in cooling is dominated by the relatively large difference in particle temperatures, which results in a weaker particle cooling for case $\mathrm{C}$ than for case $\mathrm{A}$. By increasing the size of the particle cloud in case A to $10 \mathrm{~m}$ the dashed-triple-dotted line show that the radiative cooling approaches zero, while in the right panel of Fig. 5 it is seen that the temperature approaches the situation where radiative cooling is neglected (as represented by the long-dashed black line). This is as expected since in a 

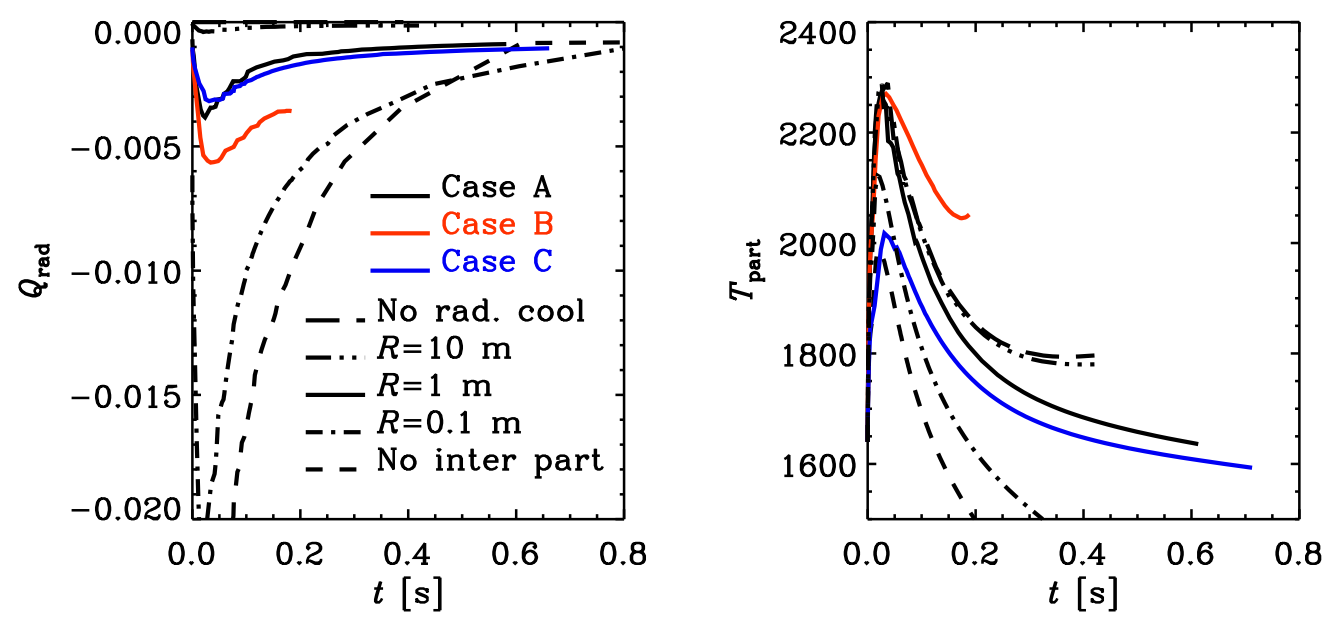

Figure 5: Radiative particle cooling, $Q_{\text {rad }}$, (left panel) and particle temperature, $T_{\text {part }}$ (right panel) as a function of time. In the left panel the line label "No inter part." correspond to the situation where inter particle radiation is neglected, i.e. where $Q_{\mathrm{rad}}$ is given by Eq. (23).

very large cloud of particles, where the optical depth is large, the volume-tosurface ratio becomes large and consequently the radiative cooling decreases. Inter-particle radiation is neglected in the traditional radiative cooling term, which is a good approximation in the limit of low optical depth, i.e. when the radius of the cloud is small. By decreasing the cloud radius to $R=0.1 \mathrm{~m}$, the radiative cooling is indeed seen to increase drastically and approaches the cooling found when the traditional radiative cooling term, as given by Eq. (23), is used.

\section{Summary}

In this work, a detailed multi-physics model for char conversion has been developed. The model allows for a detailed heterogeneous reaction mechanism and tracks species concentrations at the particle's outer surface in time. The apparent density and diameter of the particle vary with mass loss depending on the extent to which the reactive gases penetrate the particle, as determined by the effectiveness factor. The model accounts for the effects of an entire cloud of particles during gasification, as opposed to a single particle. This feature is important because it shows the impact of the entire 
cloud of particles on the gas phase temperature and composition during different stages of conversion, as well as how the cloud of particles can affect the radiative cooling. The model incorporates an effectiveness factor that is used both to determine the rate of reactions and the mode of conversion. In addition to its applicability as a stand-alone model, the presented model is also ideal as a sub model for reacting particles in a CFD simulation tool.

In the case study discussed (dry gasification of char, where the initial gas mixture consists of $84 \% \mathrm{CO}_{2}$ and $16 \% \mathrm{O}_{2}+\mathrm{N}_{2}$ ), all of the aforementioned effects were shown to significantly impact the gasification characteristics and char conversion time. Because of the incorporation of the entire particle cloud, it is seen that when keeping the initial gas composition unchanged, a $33 \%$ denser char particle cloud can result in a large increase in effectiveness factor by the late stages of conversion, thereby changing the gasification from a zone II regime (for the less dense cloud) to a zone I regime (for the more dense cloud). If the equivalence ratio is kept constant when changing the particle number density of the cloud, it is found that the char conversion time and mode of conversion do not change much. The particle temperature and CO yield is, however, found to be strongly dependent on the density of the cloud.

\section{Acknowledgments}

This work forms part of the CAMPS project supported by the Research Council of Norway (215707). The work has additionally been produced with support from the BIGCCS Centre, performed under the Norwegian Research Program Centres for Environment-Friendly Energy Research (FME). The authors acknowledge the following partners for their contributions: Aker Solutions, ConocoPhillips, Gassco, Shell, Statoil, TOTAL, GDF SUEZ and the Research Council of Norway (193816/S60). NELH also acknowledges the Research Council of Norway under the FRINATEK grant 231444

The research leading to these results has received funding from the PolishNorwegian Research Programme operated by the National Centre for Research and Development under the Norwegian Financial Mechanism 20092014 in the frame of Project Contract No Pol-Nor/232738/101/2014

MBT acknowledges the United States Department of Energy under award number DE-FC26-10FE0005372. 


\section{References}

[1] E. V. Samuilov, M. F. Faminskaya and E. S. Golovina, Combust. Explo. Shock Waves 40, 77 (2004).

[2] H. W. Haynes, AIChE J. 28, 517 (1982).

[3] B. Srinivas and N. E. Amundson, AIChE J. 26, 487 (1980).

[4] L. Qiao, J. Xu, A. Sane and J. Gore, Combustion and Flame 159, 1693 (2012).

[5] T. Govind and J. Shah, AIChE Journal 30, 79 (1984).

[6] Q. Ni and A. Williams, Fuel 74, 102 (1995).

[7] D. Vamvuka, E. T. Woodburn and P. R. Senior, Fuel 74, 1452 (1995).

[8] G.-S. Liu, G. R. Rezaei, J. A. Lucas, D. J. Harris and T. F. Wall, Fuel 79, 1767 (2000).

[9] B. Haynes Combustion \& Flame, 126, 1421-1432 (2001)

[10] B. R. Alexander, R. E. Mitchell and T. M. Gür, Journal of the Electrochemical Society 159, B347 (2012).

[11] A. C. Lee, R. E. Mitchell and T. M. Gür, AIChE J. 55, 983 (2009).

[12] M. Tilghman and R. E. Mitchell, "Coal and Biomass Char Reactivities in Gasification and Combustion Environments", Submitted for publication in Combustion \& Flame, May 2014

[13] E. S. Hecht, C. R. Shaddix, A. Molina and B. S. Haynes, Proceedings of the combustion institute 33, 1699 (2011).

[14] R. E. Mitchell, L. Ma and B-J Kim, Combustion and Flame 151, 426 (2007).

[15] R. E. Mitchell, Gasification characteristics of coal/biomass mixed fuels, Final Report, USDOE/NETL Award No. DE-FC26-10FE0005372, 2014.

[16] Y. C. Guo, C. K. Chan and K. S. Lau, Fuel 82, 893 (2003). 
[17] D. A. Frank-Kamenetskii, Diffusion and heat transfer in Chemical Kinetics. Plenum Press, New York (1969).

[18] S. K. Bhatia and D. D. Perlmutter, AIChE J. 26, 379 (1980).

[19] A. Wheeler, Advances in Catalysis 3, 249 (1951).

[20] E. W. Thiele, Ind. Eng. Chem 31, 916 (1939).

[21] N. E. L. Haugen, M. Tilghman and R. E. Mitchell, Combustion and Flame 161, 612 (2014).

[22] N. E. L. Haugen and R. E. Mitchell, "Modeling radiation in particle clouds: On the importance of inter-particle radiation for pulverized solid fuel combustion", Submitted for publication in Heat and Mass Transfer (preprint is available at http://arxiv.org/abs/1404.6587)

[23] M. C. Ma, T. C. Brown and B. S. Haynes, Surface Science 297, 312 (1993).

[24] Du, Z., "Kinetic Modeling of Carbon Oxidation," Ph.D. Thesis, Massachusetts Institute of Technology, Cambridge, MA, 1990. 